PROCEEDINGS OF THE

AMERICAN MATHEMATICAL SOCIETY

Volume 126, Number 2, February 1998, Pages 353-356

S $0002-9939(98) 04128-8$

\title{
A NOTE ON THE COHOMOLOGY OF FINITARY MODULES
}

\author{
U. MEIERFRANKENFELD
}

(Communicated by Ronald M. Solomon)

\begin{abstract}
Let $G$ be a group, $D$ a division ring and $V$ a $D G$-module. $V$ is called finitary provided that $V / C_{V}(g)$ is finite dimensional for all $g \in G$. We investigate the first and second degree cohomology of finitary modules in terms of a local system for $G$.
\end{abstract}

In this note we prove the following three theorems on the cohomology of finitary modules in terms of the cohomology of a local system of subgroups:

Theorem 1. Let $G$ be a group, $K$ a field, $V$ a finitary $K G$-module and $\mathcal{L}$ a local system of subgroups of $G$. Suppose that, for all $H \in \mathcal{L}, V$ is completely reducible as a $K H$-module. Then $[V, G]$ is completely reducible as a $K G$-module.

Theorem 2. Let $G$ be a group, $D$ a division ring, $V$ a finitary $D G$-module, $\mathcal{L}$ a local system of subgroups of $G$ and $H$ an extension of $V$ by $G$ (i.e. $H / V \cong G$ ). Suppose that the following holds for all $L$ in $\mathcal{L}$ :

(i) The extension of $V$ by $L$ in $H$ splits.

(ii) $V / C_{V}(L)$ is finite dimensional.

(iii) $H^{1}(L, V)$ is finite dimensional.

Then $H$ splits over $V$.

Theorem 3. Let $G$ be a group, $D$ a division ring, $\mathcal{L}$ a local system of subgroups of $G, W$ a $D G$-module and $V$ a $D G$-submodule of $W$ such that $W=V+C_{W}(H)$ for all $H \in \mathcal{L}$. Then there exists a canonical $D G$-monomorphism from $W / C_{W}(G)$ to $\left[V^{*}, G\right]^{*}$, where $Y^{*}$ denotes the dual of a module $Y$.

We remark that conditions (ii) and (iii) in Theorem 2 are automatically fulfilled if all members of $\mathcal{L}$ are finite groups generated by elements whose order is coprime to the characteristic of $D$.

Proof of Theorem 1. Let $H \in \mathcal{L}$. Then $[V, H]=[V, H, H]$ and so $[V, G]=[V, G, G]$. Hence we may assume that $V=[V, G]$. Let $W$ be the sum of all the irreducible $K G$-submodules in $V$, where $W=0$ if $G$ has no irreducible submodules in $V$. We need to show that $W=V$.

So suppose that $V \neq W$. Then $[V, G] \not \leq W$ and we may assume that $[V, H] \not \leq W$ for all $H \in \mathcal{L}$. Let $H \in \mathcal{L}$ and let $I_{H}$ be the set of irreducible $K H$-submodules $I$ in $[V, H]$ with $I \not \leq W$. For $I \in I_{H}$ let $m(I)$ be the supremum of all positive integers $t$ such that $I^{t}$ is isomorphic to a $K H$-submodule of $V$. Pick $h \in H$ with

Received by the editors August 20, 1996.

1991 Mathematics Subject Classification. Primary $20 J 05$.

(C)1998 American Mathematical Society 
$[I, h] \neq 0$. Then $m(I) \cdot \operatorname{deg}_{I}(h) \leq \operatorname{deg}_{V}(h)$. In particular, $m(I)$ is finite. Note that there exists a unique $K H$-submodule $\hat{I}$ in $V$ isomorphic to $I^{m(I)}$, namely $\hat{I}$ is the submodule generated by all the $H$-submodules in $V$ isomorphic to $I$. Let $K(I)=$ $\operatorname{Hom}_{K H}(I, I)$ and $d(I)=\operatorname{dim}_{K} K(I) . \operatorname{Since}_{\operatorname{dim}_{K}}[I, h]=\operatorname{dim}_{K(I)}[I, h] \cdot \operatorname{dim}_{K} K(I)$, $d(I) \leq \operatorname{deg}_{V}(h)$ and so $d(I)$ is finite. Let $m$ be the minimum of all $m(I), I \in I_{H}$, $H \in \mathcal{L}$, and $d$ the minimum of all $d(I), I \in I_{H}, H \in \mathcal{L}, m(I)=m$.

Pick $H \in \mathcal{L}$ and $I \in I_{H}$ with $m(I)=m$ and $d(I)=d$. Without loss $H \leq F$ for all $F \in \mathcal{L}$. Let $F \in \mathcal{L}$. Since $V$ is completely reducible as a $K F$-module, there exists $J \in I_{F}$ such that $I$ is isomorphic to a $K H$-submodule of $J$. Let $a$ be a positive integer such that $I^{a}$ is isomorphic to a $K H$-submodule of $J$. Then $I^{a \cdot m(J)}$ is isomorphic to a $K H$-submodule of $V$ and so $a \cdot m(J) \leq m$. By minimal choice of $m, m \leq m(J)$. Thus $a=1$ and $m(J)=m$. In particular, $\hat{I} \leq \hat{J}$ and there exists a unique $K H$-submodule $U$ in $J$ isomorphic to $I$. Hence $K(J)$ acts on $U$ and the restriction $\left.K(J)\right|_{U}$ of $K(J)$ to $U$ is contained in $K(U)$. Since $\operatorname{dim}_{K} K(U)=\operatorname{dim}_{K} K(I)=d \leq \operatorname{dim}_{K} K(J)$, we conclude that $\left.K(J)\right|_{U}=K(U)$. It is now easy to see that every irreducible $K H$-submodule of $\hat{I}$ lies in an irreducible $K F$-submodule of $\hat{J}$. Hence $\left\langle I^{F}\right\rangle$ is an irreducible $K F$-module for all $F \in \mathcal{L}$ and $\left\langle I^{G}\right\rangle$ is an irreducible $K G$-submodule in $V$ not contained in $W$. This contradiction completes the proof of Theorem 1.

The following definition and lemma are used in the proof of Theorem 2.

Definition 4. (a) Let $R$ be a ring, $A$ a set, $M$ an $R$-module and for $a \in A$ let $\rho_{a}: A \rightarrow M$ be a bijection. Then $A$ is called an affine $R$-module provided that for all $a, b, c$ in $A, \rho_{a}(b)+\rho_{b}(c)=\rho_{a}(c)$.

(b) Let $R$ be a ring, $A$ and $B$ affine $R$-modules and $\pi: A \rightarrow B$. Then $\pi$ is called an affine $R$-homomorphism if for some $a$ in $A$ and $b$ in $B, \rho_{b} \pi \rho_{a}^{-1}$ is an $R$-homomorphism of modules.

(c) Let $R$ be a ring and $A$ an affine $R$-module. A subset $B$ of $A$ is called an affine $R$-submodule if $\rho_{a}(B)$ is an $R$-submodule of $M$ for some $a$ in $A$.

Remark. Let $M$ be an $R$-module and define $\rho_{x}: M \rightarrow M, y \rightarrow y-x$. Then $M$ is an affine $R$-module. Moreover, if $A$ is any affine $R$-module with $M$ as underlying module, then for all $a$ in $A, \rho_{a}$ is an isomorphism of affine $R$-modules. Finally if $a, b$ are in $A$ and $C$ is a subset of $A$, then $\rho_{a}(C)=\rho_{b}(C)+\rho_{a}(b)$ and so $C$ is an affine submodule if and only if $\rho_{a}(C)$ is the coset of an $R$-submodule in $M$.

Lemma 5. Let $G$ be a group, $R$ a ring and $V$ an $R G$-module. Let $A_{G}$ be the set of complements to $V$ in $V \rtimes G$. Then

(a) $A_{G}$ is an affine $R$-module.

(b) Let $H \leq G$. Then the canonical map from $A_{G}$ to $A_{H}$ is affine.

(c) Let $I_{G}=\left\{G^{v} \mid v \in V\right\}$. Then $I_{G}$ is an affine $R G$ submodule of $A_{G}, I_{G} \cong$ $V / C_{V}(G)$ and $A_{G} / I_{G} \cong H^{1}(G, V)$.

Proof of the lemma. Identify $V$ and $G$ with their images in the semidirect product $V \rtimes G$. So $V \rtimes G=V G$.

(a) Let $M_{G}$ be the set of functions $f: V G / V \rightarrow V$ with $f(a b)=f(a)^{b^{-1}}+f(b)$ for all $a, b$ in $V G / V$, i.e. $M_{G}$ is the set of derivations for $G$ on $V$. Note that $M_{G}$ is an $R$-module via $(r \cdot f)(a)=r \cdot f(a)$. For $K, L$ in $A_{G}$ define $\rho_{K}(L) \in M_{G}$ by $\rho_{K}(L)(V a)=v$, whenever $a \in K$ and $v \in V$ with $v a \in L$. Then $\rho_{K}$ is a bijection from $A_{G}$ onto $M_{G}$ (see for example [As, 17.1]). 
Let $K, L, N$ be in $A_{G}$ and $a$ in $K$. Put $b=\rho_{K}(L)(V a) a$ and $c=\rho_{L}(N)(V b) b$. Then $V a=V b=V c, b \in L, c \in N$ and $c=\rho_{L}(N)(V a) \rho_{K}(L)(V a) a$. Thus $\rho_{K}(L)+\rho_{L}(N)=\rho_{K}(N)$. (Here we write the binary operation on $V$ multiplicatively whenever $V$ is regarded as a subgroup of $V \rtimes G$.)

(b) For $L$ in $A_{G}$ let $\pi(L)=L \cap V H$. Then it is easy to check that $\rho_{H} \pi \rho_{G}^{-1}$ is just the restriction map $M_{G} \rightarrow M_{H}, \phi \rightarrow \phi_{V H / V}$. Thus $\pi$ is affine.

(c) Define $\alpha: V \rightarrow M$ by $\alpha(v)(a)=v^{a}-v$. Then $\operatorname{ker} \alpha=C_{V}(G)$ and $\alpha(V)=$ $\rho_{G}\left(I_{G}\right)$ is the set of inner derivations. In particular $H^{1}(G, V)=M / \alpha(V) \cong A_{G} / I_{G}$ and (c) holds.

Proof of Theorem 2. Let $L \in \mathcal{L}$. By (i) we may view $V \rtimes L$ as a subgroup of $H$ and by part (a) of the Lemma, $A_{L}$ is an affine $D$-module and by (ii), (iii) and part (c) of the Lemma, $A_{L}$ is finite dimensional. For $L$ and $K$ in $\mathcal{L}$ with $L \leq K$ let $\pi_{K, L}$ be the affine map defined in part (b) of the Lemma. We claim that the inverse limit of $\left(\pi_{K, L}\right)_{L \leq K}$ is not empty. Note that finite dimensional affine $D$ modules fulfill the descending chain condition on affine subspaces and so a set of affine subspaces whose intersection is empty has a finite subset whose intersection is empty. Moreover, images and inverse images of affine subspaces under affine maps are affine. Now the proof in $[\mathrm{KW}, 1 \mathrm{~K} 1]$ that inverse limits of non-empty finite sets are not empty carries over word for word, except that "subset" has to be replaced by "affine subspace". Let $\left(C_{L}\right)_{L \in \Lambda}$ be an element in the inverse limit. Then $\bigcup\left\{C_{L} \mid L \in \mathcal{L}\right\}$ is a complement to $V$ in $H$ and Theorem 2 is proved.

Proof of Theorem 3. For $X \leq V^{*}$ let $X^{\perp}=\{v \in V \mid x(v)=0$ for all $x \in X\}$. We will first prove that:

$$
\text { For all } K \leq G,\left[V^{*}, K\right]^{\perp}=C_{V}(K) .
$$

Indeed, let $x \in V^{*}, k \in K$ and $v \in V$. Then

$$
[x, k](v)=\left(x^{k}-x\right)(v)=x^{k}(v)-x(v)=x\left(v^{k^{-1}}\right)-x(v)=x\left(\left[v, k^{-1}\right]\right) .
$$

It follows that $v \in\left[V^{*}, K\right]^{\perp}$ if and only if $[v, K] \leq V^{* \perp}=0$ and so if and only if $v \in C_{V}(K)$.

Let $H \in \mathcal{L}$. Define a map $a_{H}: W \rightarrow\left[V^{*}, H\right]^{*}$ by $a_{H}(w)(x)=x(u)$ where $x \in\left[V^{*}, H\right], w \in W$ and $u \in V$ with $w \in u+C_{W}(H)$. Note that by $(*)$ this definition does not depend on the choice of $u$. If $K \leq H$ with $K \in \mathcal{L}$, then $C_{W}(H) \leq C_{W}(K)$ and so $w \in u+C_{W}(K)$ and $a_{H}(w)(x)=a_{K}(w)(x)$ for all $x \in\left[V^{*}, K\right]$. Define $a: W \rightarrow\left[V^{*}, G\right]^{*}$ by $a(w)(x)=a_{H}(w)(x)$ whenever $w \in W$, $x \in\left[V^{*}, G\right]$ and $H \in \mathcal{L}$ with $x \in\left[V^{*}, H\right]$. By the preceding observation and since $\mathcal{L}$ is a local system this definition does not depend on the choice of $H$. Let $w \in W$ with $a(w)=0$. Then $a_{H}(w)=0$ for all $H \in \mathcal{L}$ and so $u \in\left[V^{*}, H\right]^{\perp}$, where $u$ is as above. By $(*), u \in C_{V}(H)$ and so $w \in C_{W}(H)$ for all $H \in \mathcal{L}$. Thus $\operatorname{ker} a=C_{W}(G)$. It remains to show that $a$ is a $D G$-homomorphism. Clearly $a$ is $D$-linear. Let $w, x, u$ and $H$ be as above and $g \in G$. We may assume without loss that $g \in H$. Then $w^{g} \in u^{g}+C_{W}(H)$ and so

$$
\begin{aligned}
a\left(w^{g}\right)(x) & =a_{H}\left(w^{g}\right)(x)=x\left(u^{g}\right)=x^{g^{-1}}(u) \\
& =a_{H}(w)\left(x^{g^{-1}}\right)=a(w)\left(x^{g^{-1}}\right)=a(w)^{g}(x) .
\end{aligned}
$$

Thus $a\left(w^{g}\right)=a(w)^{g}$ and $a$ is a $D G$-homomorphism, completing the proof of Theorem 3. 


\section{REFERENCES}

[As] M. Aschbacher, Finite Group Theory, Cambridge University Press, Cambridge (1986). MR 89b:20001; MR 95b:20002

[KW] O. Kegel and B. A. F. Wehrfritz, Locally Finite Groups, North Holland (1973). MR 57:9848

Department of Mathematics, Michigan State University, East Lansing, Michigan 48824

E-mail address: meier@math.msu.edu 